Proyecciones Journal of Mathematics

Vol. 32, $\mathrm{N}^{\circ}$ 4, pp. 359-375, December 2013.

Universidad Católica del Norte

Antofagasta - Chile

\title{
Banach's and Kannan's fixed point results in fuzzy 2-metric spaces
}

\author{
Binod Chandra Tripathy \\ Institution of Advanced Study in Sc. and Tech., India \\ Sudipta Paul and Nanda Ram Das \\ Gauhati University, India \\ Received: August 2013. Accepted : September 2013
}

\begin{abstract}
In this paper we establish two common fixed point theorems in fuzzy 2- metric spaces. These theorems are generalizations of the Banach Contraction mapping principle and the Kannan's fixed point theorem respectively in fuzzy 2-metric spaces.
\end{abstract}

AMS Classification(2000): 47H10, 54H25

Keyword and phrases: Fuzzy 2-metric space, Hadzic type $t$ norm, weakly compatible mapping, $\psi$-function. 


\section{Introduction}

L. A. Zadeh introduced the notion of fuzzy sets in 1965. The importance of the introduced notion of fuzzy set was realized and has successfully been applied in almost all the branches of science and technology. Recently fuzzy set theory has been applied in introducing different classes of sequences and for investigating their different properties by Tripathy and Baruah [11], Tripathy, Baruah, Et and Gungor [12], Tripathy and Borgohain [13], Tripathy and Dutta [14], Tripathy and Sarma [16]. Fuzzy topological spaces has been investigated by Tripathy and Debnath [14], Tripathy and Ray [17] and many others.

Kramosil and Michlek [10] introduced the concept of fuzzy metric space in 1975 and fixed point theorems for fuzzy metric space was first obtained by Heipern [9]. Later George and Veeramani [7] modified the notion of fuzzy metric space with the help of $t$-norm and defined the Hausdoff topology of fuzzy metric spaces.

Gahler ([5], [6]) investigated the properties of 2-metric space and then authors investigated contraction mapping in 2-metric spaces. The idea of fuzzy 2-metric space was used by Sharma [11] and obtained some fruitful results.

Ciric [4] has introduced a generalization of the Banach Contracion mapping principle in fuzzy metric spaces. In one theorem we have extended the result of Ciric [4] to coincidence point theorem of three mappings. In another theorem we have proved a fixed point result for generalized Kannan type mapping.

\section{Preliminaries and definitions}

Definition 2.1. An operation $*:[0,1] \times[0,1] \times[0,1] \rightarrow[0,1]$ is called a continuous $t$-norm if $([0,1], *)$ is an abelian topological monoid with the unit 1 such that for all $a, b, c, d, e, f \in[0,1]$

$a * b * c \leq d * e * f$ whenever $a \leq d, b \leq e, c \leq f$.

The following are some examples of $t$-norm. 
Banach's and Kannan's fixed point results in fuzzy 2-metric spaces 361

Example 2.1. Minimum $t$-norm $\left(*_{M}\right): *_{M}(x, y)=\min \{x, y\}$.

Example 2.2. Product $t$-norm $\left(*_{P}\right): *_{P}(x, y)=x . y$.

Example 2.3. Lukasiewicz $t$-norm $\left(*_{L}\right): *_{L}(x, y)=\max \{x+y-1,0\}$.

Definition 2.2. The 3 -tuple $(X, M, *)$ is called a fuzzy 2-metric space if $X$ is an arbitrary set, ${ }^{*}$ is a continuous $t$-norm and $M$ is a fuzzy set in $X^{3} \times[0, \infty)$ satisfying the following conditions for all $x, y, z, u \in X$ and $t_{1}, t_{2}, t_{3}>0$.

(1) $M(x, y, z, 0)=0$.

(2) $M(x, y, z, t)=1$ for all $t>0$ when at least two of the three points are equal.

(3) $M(x, y, z, t)=M(x, z, y, t)=M(y, z, x, t)$ (Symmetry about three variables).

(4) $M\left(x, y, z, t_{1}+t_{2}+t_{3}\right) \geq M\left(x, y, u, t_{1}\right) * M\left(x, u, z, t_{2}\right) *\left(M\left(u, y, z, t_{3}\right)\right.$. (This corresponds to tetrahedron inequality in 2-metric spaces).

(5) $M(x, y, z,):.[0, \infty) \rightarrow[0,1]$ is left continuous.

(6) $\lim _{t \rightarrow \infty} M(x, y, z, t)=1$ for all $x, y, z \in X$ and $t>0$.

Lemma 2.3. Let $(X, M, *)$ be a fuzzy 2 -metric space. Then $M(x, y, z,$. is non-decreasing function for all $x, y, z \in X$.

Proof. Suppose $M(x, y, z, t)>M(x, y, z, s)$ for same $s, t>0$ with $s>t$.

Then we have,

$M(x, y, z, t)$

$>M(x, y, z, s)$

$\geq M\left(x, y, y, \frac{s}{2}+\frac{t}{2}\right) * M(x, y, z, t) * M\left(y, y, z, \frac{s}{2}-\frac{t}{2}\right)$

$\geq 1 * M(x, y, z, t) * 1$ 
$=M(x, y, z, t)$

which is a contradiction.

Hence $M(x, y, z,$.$) in non-decreasing.$

Definition 2.4. A sequence $\left\{x_{n}\right\}$ in a fuzzy 2-metric space $(X, M, *)$ is said to converge to $x$ in $X$ if and only if $\lim _{n \rightarrow \infty} M\left(x_{n}, x, a, t\right)=1$ for all $a \in x$ and $t>0$.

Definition 2.5. Let $(X, M, *)$ be a fuzzy 2-metric space. A sequence $\left\{x_{n}\right\}$ in $X$ is called Cauchy sequence, if and only if

$$
\lim _{n \rightarrow \infty} M\left(x_{n+p}, x_{n}, a, t\right)=1 \text { for all } a \in X \text { and } p>0, t>0 .
$$

Definition 2.6. A fuzzy 2-metric space $(X, M, *)$ is said to be complete if and only if every Cauchy sequence in $X$ is convergence in $X$.

The following definition is given by Hadzic and Pap [7].

Definition 2.7. A $t$-norm * is said to be Hadzic type $t$-norm if the family $\left\{*^{p}\right\}_{p \in N}$ of its iterates defined for each $s \in(0,1)$ by $*^{0}(s)=1, *^{(p+1)}(s)=$ $*\left(*^{p}(s), s\right)$ for all $p>0$ is equi-continuous at $\mathrm{s}=1$, that is, given $\lambda>0$, there exists $\eta(\lambda) \in(0,1)$ such that $1 \geq s>\eta(\lambda) \Rightarrow *(p)_{(s)}>1-\lambda$ for all $p \in N$.

Lemma 2.8. Let $(X, M, *)$ be a fuzzy 2 -metric space such that $M(x, y, a, t) \rightarrow 1$ as $t \rightarrow \infty$ for all $x, y \in X$, where $*$ is a Hadzic type $t$-norm. If the sequence $\left\{x_{n}\right\}$ in $X$ is such that for all $n \in N$,

$$
M\left(x_{n}, x_{n+1}, a, t\right) \geq M\left(x_{n-1}, x_{n}, a, t / k\right),
$$

where $0<k<1, t>0$, then the sequence $\left\{x_{n}\right\}$ is a Cauchy sequence.

Lemma 2.9. $M$ is a continuous function on $X^{3} \times(0, \infty)$.

Definition 2.10. Two mappings $f, g: X \rightarrow X$, where $X$ is a nonempty set, are said to be weakly compatible if they commute at their coincidence point, that is for any $x \in X, f x=g x$ implies that $f g x=g f x$. 
Definition 2.11. ( $\Psi$-function). A function $\psi:[0,1] \times[0,1] \rightarrow[0,1]$ is said to be a $\Psi$-function if

(1) $\psi$ is monotonic increasing and continuous.

(2) $\psi(t, t) \geq t$ for all $0 \leq t \leq 1$.

Example 2.4. Some examples of $\Psi$-function are,

(a) $\psi(x, y)=\sqrt{x y}$.

(b) $\psi(x, y)=\min \{x, y\}$, where $x, y \in[0.1]$.

\section{Main results}

Theorem 3.1. Let $(X, M, *)$ be a fuzzy 2 -metric space such that

$M(x, y, a, t) \rightarrow 1$ as $t \rightarrow \infty$ for all $x, y \in X$, where $*$ is a Hadzic type $t$-norm and let $A, B, g: X \rightarrow X$ be three mappings such that,

(i) $g X$ is closed.

(ii) $A X \subseteq g X$ and $B X \subseteq g X$.

(iii) $\mathrm{M}(\mathrm{Ax}, \mathrm{By}, \mathrm{a}, \mathrm{kt})+\mathrm{q}(1-\max (\mathrm{M}(\mathrm{gx}, \mathrm{By}, \mathrm{a}, \mathrm{kt}), \mathrm{M}(\mathrm{gy}, \mathrm{Ax}, \mathrm{a}, \mathrm{kt})))$

$$
\geq M(g x, g y, a, t)
$$

where $x, y \in X, x \neq y, t>0$ and $0<k<1$.

Then mappings $A, B$ and $g$ have a coincidence point.

Proof. Let $x_{0} \in X$ be an arbitrary point. A sequence $\left\{x_{n}\right\} \in X$ is constructed by $g x_{1}=A x_{0}, g x_{2}=B x_{1}, g x_{3}=A x_{2}$ for all $n \in N$,

$$
g x_{2 n-1}=A x_{2 n-2}, g x_{2 n}=B x_{2 n-1} .
$$

This is possible by condition ( $i i)$ of the theorem. If $x_{n}=x_{n+1}$ for some $n$, then the proof of the theorem is trivial. So let $x_{n} \neq x_{n+1}$ for all $n \in N$. 
Now, putting $x=x_{2 n}$, and $y=x_{2 n+1}$ in (3.1), for all $t>0$,

We have,

$$
\begin{aligned}
& M\left(A x_{2 n}, B x_{2 n+1}, a, k t\right)+q\left(1-\max M\left(g x_{2 n}, B x_{2 n+1}, a, k t\right),\right. \\
& \left.\mathrm{M}\left(\mathrm{gx}_{2 n+1}, A x_{2 n}, a, k t\right)\right) \\
& \geq M\left(g x_{2 n}, g x_{2 n+1}, a, t\right) \\
& \Rightarrow M\left(g x_{2 n+1}, g x_{2 n+2}, a, k t\right)+q\left(1-\max M\left(g x_{2 n}, g x_{2 n+2}, a, k t\right),\right. \\
& \left.\mathrm{M}\left(\mathrm{gx}_{2 n+1}, g x_{2 n+1}, a, k t\right)\right) \\
& \geq M\left(g x_{2 n}, g x_{2 n+1}, a, t\right) \\
& \Rightarrow M\left(g x_{2 n+1}, g x_{2 n+2}, a, k t\right)+q(1-1) \\
& \geq M\left(g x_{n}, g x_{2 n+1}, a, t\right) .
\end{aligned}
$$

Hence, for all $t>0 ; n \geq 0$, we have

$$
M\left(g x_{2 n+1}, g x_{2 n+2}, a, k t\right) \geq M\left(g x_{2 n}, g x_{2 n+1}, a, t\right) .
$$

On considering, $x=x_{2 n}$ and $y=x_{2 n-1}$ in (3.1), for all $t>0$, we have,

$M\left(A x_{2 n}, B x_{2 n-1}, a, k t\right)+q\left(1-\max M\left(g x_{2 n}, B x_{2 n-1}, a, k t\right)\right.$, $\left.\mathrm{M}\left(\mathrm{gn}_{2 n-1}, A x_{2 n}, a, k t\right)\right)$.

$\geq M\left(g x_{2 n}, g x_{2 n-1}, a, t\right)$.

$\Rightarrow M\left(g x_{2 n+1}, g x_{2 n}, a, k t\right)+q\left(1-\max M\left(g x_{2 n}, g x_{2 n}, a, k t\right)\right.$

$\left.\mathrm{M}\left(\mathrm{gx}_{2 n-1}, g x_{2 n+1}, a, k t\right)\right)$.

$\geq M\left(g x_{2 n}, g x_{2 n-1}, a, t\right)$.

$\Rightarrow M\left(g x_{2 n+1}, g x_{2 n}, a, k t\right)+q(1-1) \geq M\left(g x_{2 n}, g x_{2 n-1}, a, t\right)$.

Thus, for all $t>0, n \in N$, we have, 
Banach's and Kannan's fixed point results in fuzzy 2-metric spaces 365

$$
M\left(g x_{2 n+1}, g x_{2 n}, a, k t\right) \geq M\left(g x_{2 n}, g x_{2 n-1}, a, t\right) .
$$

From, (3.2) and (3.3), for all $n \geq 0$ and $t>0$, we have

$$
M\left(g x_{n}, g x_{n+1}, a, k t\right) \geq M\left(g x_{n-1}, g x_{n}, a, t\right)
$$

Then, from Lemma 2.8, we conclude that $g x_{n}$ is a Cauchy sequence. Since, $g X$ is closed, there exists $n \in X$ Such that,

$$
\lim _{n \rightarrow \infty} g x_{n}=g x .
$$

From (3.4) and the fact that $x_{n} \neq x_{n+1}$ for all $n \in N$, without loss of generality, we assume that $x_{n} \neq x$ for all $n \in N$, otherwise there exists a subsequence with this property.

Putting $x=x_{2 n}$ and $y=x$ in (3.1), for all $t>0$ and $n \in N$, we have $M\left(A x_{2 n}, B x, a, k t\right)+q\left(1-\max M\left(g x_{2 n}, B x, a, k t\right), M\left(g x, g x_{2 n+1}, a, k t\right)\right.$ $\geq M(g x 2 n, g x, a, t)$.

Taking $n \rightarrow \infty$ on both sides of the above inequality, by Lemma 2.9 and using (3.4), we have

$$
\lim _{n \rightarrow \infty} M\left(A x_{2 n}, B x, a, k t\right)=1 .
$$

Now, for all $t>0$ and $n \in N$, we have

$$
\begin{aligned}
& M(g x, B x, a, t) \\
& >M\left(g x, g x_{2 n+1}, a, t-k t\right) *\left(M\left(g x, B x, g x_{2 n+1}, k t / 2\right) * M\left(g x_{2 n+1}, B x, a, k t / 2\right)\right. \\
& =M\left(g x, g x_{2 n+1}, a, t-k t\right) * M(g x, B x, A x 2 n, k t / 2) * M\left(A x_{2 n}, B x, a, k t\right) .
\end{aligned}
$$

Taking $n \rightarrow \infty$ in the above inequality from (3.4) and (3.5), we have for all $t>0, M(g x, B x, a, t)=1$, which implies that

$$
B x=g x .
$$


Putting $x=x$ and $y=x_{2 n-1}$ in (3.1), for all $t>0$, we have

$M\left(A x, B x_{2 n-1}, a, k t\right)+q\left(1-\max M\left(g x, g x_{2 n}, a, k t\right), M\left(g x_{2 n-1}, A x, a, k t\right)\right)$

$\geq M\left(g x, g x_{2 n-1}, a, t\right)$.

On taking $n \rightarrow \infty$ both sides of the above inequalities, by Lemma 2.9 and using (3.4) we have,

$$
\lim _{n \rightarrow \infty} M\left(A x, B x_{2 n-1}, a, k t\right)=1 .
$$

Now, for all $t>0$ and $n \in N$, we have,

$M(g x, A x, a, t)$

$\geq M\left(g x, g x_{2 n}, a, t-k t\right) * M\left(g x_{2 n}, A x, a, \frac{k t}{2}\right) * M\left(g x, A x, g x_{2 n}, \frac{k t}{2}\right)$

$=M\left(g x, g x_{2 n}, t(1-k)\right) * M\left(B x_{2 n-1}, A x, a, \frac{k t}{2}\right) * M\left(g x, A x, B x_{2 n-1}, \frac{k t}{2}\right)$

Taking $n \rightarrow \infty$ in the above inequality, using (3.4) and (3.7), we have for all $t>0, M(g x, A x, a, t)=1$, which implies that

$$
A x=g x .
$$

From (3.6) and (3.8) we conclude that

$A x=B x=g x$, that is, $x$ is a coincidence point of the mappings $A, B$ and $g$.

Theorem 3.2. Let $(X, M, *)$ be a complete fuzzy 2-metric space such that $M(x, y, a, t)$ is strictly increasing in the variable $t$ and $M(x, y, a, t) \rightarrow$ 1 as $t \rightarrow \infty$ for all $x, y \in X$, where ${ }^{*}$ is a Hadzic type $t$-norm. let $A, g: X \rightarrow X$ be two self mapping on $X$, such that the following conditions are satisfied:

(i) $g X$ is closed.

(ii) $A X \subseteq g X$. 
Banach's and Kannan's fixed point results in fuzzy 2-metric spaces 367

(iii) $M(A x, A y, a, k t)+q(1-\max M(g x, A y, a, k t), M(g y, A x, a, k t))$

$$
\geq \psi(M(g x, A x, a, t), M(g y, A y, a, t)) \text { for all } x, y \in X,
$$

where $q=q(x, y, a, t) \geq 0, t>0,0<k<1$ and $\psi$ is a $\Psi$-function.

Then $A$ and $g$ have a coincidence point. Further if $(A, g)$ is a weekly compatible pair, then $A$ and $g$ have an unique common fixed point.

Proof. Let $x_{0} \in X$ be any point. We construct a sequence $\left\{x_{n}\right\}$ as follows:

$y_{1}=g x_{1}=A x_{0}, y_{2}=g x_{2}=A x_{1}$ and in general $y_{n}=g x_{n}=A x_{n-1}$ for all $n \in N$.

This is possible by condition (ii) of the theorem.

Further we assume that $y_{n} \neq y_{n+1}$, for all $n \in N$, otherwise $g$ and $A$ have a coincidence point.

Thus, for all $t>0, n \in N$, we have

$$
0<M\left(y_{n}, y_{n+1}, a, t\right)<1 .
$$

Putting $x=x_{n}$ and $y=x_{n-1}$ in (3.9) for all $t>0$, we have

$$
\begin{aligned}
& M\left(A x_{n}, A x_{n-1}, a, k t\right)+q\left(1-\max M\left(g x_{n}, A x_{n-1}, a, k t\right), M\left(g x_{n-1}, A x_{n}, a, k t\right)\right) \\
& \geq \psi\left(M\left(g x_{n}, A x_{n}, a, t\right), M\left(g x_{n-1}, A x_{n-1}, a, t\right)\right) . \\
& \Rightarrow M\left(g x_{n+1}, g x_{n}, a, k t\right)+q\left(1-\max M\left(g x_{n}, g x_{n}, a, k t\right), M\left(g x_{n-1}, g x_{n+1}, a, k t\right)\right) \\
& \geq \psi\left(M\left(g x_{n}, g x_{n+1}, a, t\right), M\left(g x_{n-1}, g x_{n}, a, t\right)\right) . \\
& \Rightarrow M\left(g x_{n+1}, g x_{n}, a, k t\right)+q(1-1) \\
& \geq \psi\left(M\left(g x_{n}, g x_{n+1}, a, t\right), M\left(g x_{n-1}, g x_{n}, a, t\right) .\right. \\
& \Rightarrow M\left(y_{n+1}, y_{n}, a, k t\right) \geq\left(M\left(y_{n}, y_{n+1}, a, t\right), M\left(y_{n-1}, y_{n}, a, t\right) .\right.
\end{aligned}
$$


If $M\left(y_{n-1}, y_{n}, a, s\right)>M\left(y_{n}, y_{n+1}, a, s\right)$ for some $s>0$, from the above inequality, using properties of $y$ and (3.10), we obtain

$$
\begin{aligned}
& M\left(y_{n+1}, y_{n}, a, k s\right) \geq \psi\left(M\left(y_{n}, y_{n+1}, a, s\right), M\left(y_{n+1}, y_{n}, a, s\right)\right. \\
& \geq\left(M\left(y_{n}, y_{n+1}, a, s\right)\right.
\end{aligned}
$$

Hence a contradiction

Thus, for all $n \in N$ and $t>0$, we have,

$$
M\left(y_{n}, y_{n+1}, a, k t\right) \geq M\left(y_{n-1}, y_{n}, a, t\right) .
$$

Then from Lemma 2.8 we conclude that $\left\{y_{n}\right\}$ is a Cauchy sequence. Since $X$ is complete, then there exists $z \in X$. such that $\lim _{n \rightarrow \infty} y_{n}=z$.

Therefore,

$$
\lim _{n \rightarrow \infty} y_{n}=\lim _{n \rightarrow \infty} g x_{n}=\lim _{n \rightarrow \infty} A x_{n}=z .
$$

Since $g X$ is closed, there exists $u \in X$ such that

$$
g u=z .
$$

Putting $x=u$ and $y=x_{n-1}$ in (3.9), for all $t>0$, we have

$$
\begin{aligned}
& M\left(A u, A x_{n-1}, a, k t\right)+q\left(1-\max M\left(g u, A x_{n-1}, a, k t\right), M\left(g x_{n-1}, A u, a, k t\right)\right) \\
& \geq \psi\left(M(g u, A u, a, t), M\left(g x_{n-1}, A x_{n-1}, a, t\right) .\right.
\end{aligned}
$$

Taking $n \rightarrow \infty$ on both sides of the above inequality, for all $t>0$, we have,

$$
\begin{aligned}
& M(A u, z, a, k t)+q(1-\max M(g u, z, a, k t), M(z, A u, a, k t)) \\
& \geq \psi(M(z, A u, a, t), M(z, z, a, t) .
\end{aligned}
$$


Banach's and Kannan's fixed point results in fuzzy 2-metric spaces 369

$\Rightarrow M(A u, z, a, k t)+q 1-1) \geq \psi(M(z, A u, a, t), M(z, z, a, t))$.

Thus, for all $t>0$, using the properties of $\Psi$-function, we have

$$
\begin{gathered}
M(A u, z, a, k t) \geq \psi(M(z, A u, a, t), M(z, z, a, t) \\
\geq \psi(M(A u, z, a, t), M(A u, z, a, t) \\
\geq M(A u, z, a, t) .
\end{gathered}
$$

The above inequality implies that $A u=z$

Hence from (3.13), we have

$$
A u=g u=z .
$$

Therefore, $u$ is a coincidence point of $A$ and $g$.

Next let $(A, g)$ be a weekly compatible pair of mapping. Then from (3.14), we have $g A u=A g u$, that is

$$
g z=A z
$$

On putting $x=z, y=x_{n-1}$ in (3.9), for all $t>0$ we have

$$
\begin{aligned}
& M(A z, z, a, k t)+q(1-\max M(g z, z, a, k t), M(z, A z, a, k t)) \\
& \geq \psi(M(g z, A z, a, t), M(z, z, a, t)) . \\
& \Rightarrow M(A z, z, a, k t)+q(1-\max M(A z, z, a, k t), M(z, A z, a, k t)) \geq \psi(1-
\end{aligned}
$$
1) $=1$.

by $(3.15)$, that is, $M(A z, z, a, k t)+q(1-M(A z, z, a, k t)) \geq 1$.

Thus, for all $t>0, M(A z, z, a, k t)=1$.

Which implies that 


$$
A z=z .
$$

From (3.15) and (3.16) we have

$A z=g z=z$. So, $z$ is a fixed point of $A$ and $g$. is,

To prove the uniqueness, Let $z_{1}$ and $z_{2}$ be two distinct fixed point, that

$$
A z_{1}=g z_{1}=z_{1} \text { and } A z_{2}=g z_{2}=z_{2} .
$$

Putting $x=z_{1}$ and $y=z_{2}$ in (3.9), for all $t>0$, we have

$$
\begin{aligned}
& M\left(A z_{1}, A z_{2}, a, k t\right)+q\left(1-\max M\left(g z_{1}, A z_{2}, a, k t\right), M\left(g z_{2}, A z_{1}, a, k t\right)\right) \\
& \geq \psi\left(M\left(g z_{1}, A z_{1}, a, t\right), M\left(g z_{2}, A z_{2}, a, t\right)\right) \\
& \geq M\left(z_{1}, z_{2}, a, k t\right)+q\left(1-M\left(z_{1}, z_{2}, a, k t\right)\right) \geq \psi\left(M\left(z_{1}, z_{2}, a, t\right), M\left(z_{2}, z_{2}, a, t\right)\right) . \\
& \Rightarrow M\left(z_{1}, z_{2}, a, k t\right)+q\left(1-M\left(z_{1}, z_{2}, a, k t\right)\right) . \\
& \geq \psi(1,1)=1 \\
& \Rightarrow M\left(z_{1}, z_{2}, a, k t\right)(1-q) \geq(1-q) .
\end{aligned}
$$

Therefore, for all $t>0$, we get

$$
\begin{aligned}
& M\left(z_{1}, z_{2}, a, k t\right)=1 . \\
& \Rightarrow z_{1}=z_{2} .
\end{aligned}
$$

Hence the common fixed point is unique.

This establishes the Theorem.

Corollary 3.3. Let $(X, M, *)$ be a complete fuzzy 2-metric space such that $M(x, y, a, t)$ is strictly increasing in the variable $t$ and $M(x, y, a, t) \rightarrow 1$ as $t \rightarrow \infty$ for all $x, y \in X$, where ${ }^{*}$ is Hadzic type $t$-norm. 
Let $A: X \rightarrow X$ be a self mapping on $X$ which satisfies the following conditions for all $x, y \in X$ :

$$
M(A x, A y, a, k t)+q(1-\max M(x, A y, a, k t), M(y, A x, a, k t)) .
$$

$$
\geq \psi(M(x, A x, a, t) M(y, A y, a, t)),
$$

where $q=q(x, y, a, t) \geq 0, t>0,0<k<1$ and $\psi$ is a $\Psi$-function. Then $A$ has a unique fixed point.

Proof. on considering $g x=x$ for all $x \in X$ we can proof this by Theorem 3.2 .

Corollary 3.4. Let $(X, M, *)$ be a complete fuzzy 2-metric space such that $M(x, y, a, t)$ is strictly increasing in the variable $t$ and $M(x, y, a, t) \rightarrow 1$ as $t \rightarrow \infty$ for all $x, y \in X$.

Where ${ }^{*}$ is a Hadzic type $t$-norm.

Let $A: X \rightarrow X$ be self mapping on $X$.

Which satisfies the following inequality for all $x, y \in X$

$$
M(A x, A y, a, k t) \geq \psi(M(x, A x, a, t), M(y, A y, a, t)) .
$$

Where $t>0,0<k<1$ and $\psi$ is a $\Psi$-function, then $A$ has a unique fixed point.

Proof. Taking $q=0$ and $g x=x$ for all $x \in X$ in Theorem 3.2 we can proof this result.

Corollary 3.5. Let $(X, d)$ be a complete metric space and $A: X \rightarrow X$ be a mapping which satisfies the following inequality:

$$
d(A x, A y) \leq \frac{k}{2}[d(x, A x)+d(y, A y)]
$$

where $0<k<1, x, y \in X$. Then $A$ has a unique fixed point. 
Proof. Consider the corresponding fuzzy 2-metric space $(X, M, *)$, where

$M(x, y, a, t)=\frac{t}{t+d(x, y)}$ and $c * d=\operatorname{minc}, d$.

We prove that the inequality (3.19) implies the inequality (3.18) with

$\psi(x, y)=\min \{x, y\}$

Assume the contrary, then from (3.19) for some $t$,

$\frac{t}{t+\frac{1}{k} d(A x, A y)}<\min \left\{\frac{t}{t+d(x, A x)}, \frac{t}{t+d(y, A y)}\right\}$

that is, $t+\frac{1}{k} d(A x, A y)>t+d(x, A x)$

and $t+\frac{1}{k} d(A x, A y)>t+d(y, A)$

That is, $d(A x, A y)>\frac{k}{2}[d(x, A x)+d(y, A y)]$,

Hence a contradiction.

This completes the proof.

Example 3.1. Let $X=[0,1]$ and let $M(x, y, a, t)=e^{-\frac{|x-y|}{t}}$.

Let $*$ be a Hadzic type $t$-norm, then $(X, M, *)$ is a complete fuzzy 2 metric space Let $A, g: X \rightarrow X$ be defined as follows:

$$
A x=1
$$

and

$$
g x=(2+x) / 3
$$

where $x \in[0,1]$ and $\psi(x, y)=x y$. Then all the conditions of Theorem 3.2 are satisfied and 1 is the unique common fixed point. 


\section{References}

[1] B. S. Choudhury and K.Das, Fixed points of generalized Kannan type mapping in generalized Menger spaces, commun Korean Math, Soc. 24(4), pp. 529-537, (2009).

[2] B. S Choudhury K.Das and P.Das, Extensions of Banach's and Kannan's results in Fuzzy metric Space. Hacettepe Journal of Mathematics and Statistics, 39(1), pp. 1-9, (2009).

[3] B. S. Choudhury and A. Kundu, A common fixed point result in fuzzy metric spaces using altering distances, J. Fuzzy Math. 18 (2), pp. 517$52,(2010)$.

[4] L. Ciric, Some new results for Banach contradiction and Edelstein contractive mappings on fuzzy metric spaces, chaos solitons fractals 42(1), pp. 146-154, (2009).

[5] S. Gahler, Linear 2-normierte Raume, Math. Nachr, 28 pp. 1-43, (1964).

[6] S. Gahler, U ber 2-Banach Raume, Math. Nachr. 42, pp. 335-347, (1969).

[7] A. George and P. Veeramani, On some results in fuzzy metric spaces, Fuzzy sets and systems 64(3), pp. 395-399, (1994).

[8] O. Hadzic and E. Pap, Fixed Point Theory in Probabilistic Metric Space, Kluwer Academic Publishers, Dordrecht, (2001).

[9] S. Heipern, Fuzzy mapping and fixed point theorems, J. Maths, Anal. Appl. 83, pp. 565-569, (1981).

[10] O. Kramosil and J. Michalek: Fuzzy metric and statistical metric spaces, Kybernelika, 11, pp. 326-334, (1975).

[11] B. C. Tripathy and A. Baruah, Lacunary statistically convergent and lacunary strongly convergent generalized difference sequences of fuzzy real numbers, Kyungpook Math. J., 50(4), pp. 565-574, (2010). 
[12] B. C. Tripathy and A. Baruah, M.Et and M. Gungor, On almost statistical convergence of new type of generalized difference sequence of fuzzy numbers, Iranian Jour. Sci. Tech., Trans. A : Sci., 36 (2), pp. 147-155, (2012).

[13] B. C. Tripathy and S. Borgogain, Some classes of difference sequence spaces of fuzzy real numbers defined by Orlicz function; Adv. Fuzzy Syst., 2011, Article ID216414, 6 pages (2011).

[14] B. C. Tripathy and A. J. Dutta, Lacunary bounded variation sequence of fuzzy real numbers, Jour. Intell. Fuzzy Syst., 24 (1), pp. 185-189, (2013).

[15] B. C. Tripathy and S. Debnath, $\gamma$-open sets and $\gamma$-continuous mappings in fuzzy bitopological spaces, Jour. Intell. Fuzzy Syst., 24(3), pp. 631-635, (2013).

[16] B. C. Tripathy and G.C. Ray, On Mixed fuzzy topological spaces and countability, Soft Comput., 16 (10), pp. 1691-1695, (2012).

[17] B. C. Tripathy and B. Sarma, On I-convergent double sequences of fuzzy real numbers; Kyungpook Math. J., 52 (2), pp. 189-200, (2012).

[18] S. Sharma, On fuzzy metric space, Southeast Asian Bulletin of Mathematics, 26, pp. 133-145, (2002).

\section{Binod Chandra Tripathy}

Mathematical Science Division;

Institution of Advanced Study in Science and Technology;

Paschim Boragaon, Garchuk;

Guwahati-781035, ASSAM;

India

e-mail : tripathybc@yahoo.com; tripathybc@rediffmail.com

\section{Sudipta Paul}

Department of Mathematics,

Gauhati University,

Guwahati-781014;

ASSAM,

India

e-mail : sudiptapaul_math@rediiffmail.com 
Banach's and Kannan's fixed point results in fuzzy 2-metric spaces 375

and

Nanda Ram Das

Department of Mathematics,

Gauhati University,

Guwahati-781014;

ASSAM,

INDIA

e-mail : nrd47@yahoo.co.in 